\title{
Dissociation of Charge Transfer States and Carrier Separation in Bilayer Organic Solar Cells: A Time-Resolved Electroabsorption Spectroscopy Study
}

Andrius Devižis, ${ }^{*}, \dagger$ Jelissa De Jonghe-Risse, ${ }^{\dagger}$ Roland Hany, ${ }^{\S}$ Frank Nüesch, ${ }^{\S, \ddagger}$ Sandra Jenatsch,, , Vidmantas Gulbinas," and Jacques-E. Moser ${ }^{\dagger}$

†Photochemical Dynamics Group, Institute of Chemical Sciences and Engineering, and ${ }^{\dagger}$ Institute of Materials, École Polytechnique Fédérale de Lausanne, $\mathrm{CH}-1015$ Lausanne, Switzerland

${ }^{\S}$ Laboratory for Functional Polymers, EMPA, Swiss Federal Laboratories for Materials Science and Technology, CH-8600, Dübendorf, Switzerland

"Center for Physical Sciences and Technology, Savanoriu 231, LT-02300 Vilnius, Lithuania

Supporting Information

ABSTRACT: Ultrafast optical probing of the electric field by means of Stark effect in planar heterojunction cyanine dye/fullerene organic solar cells enables one to directly monitor the dynamics of free electron formation during the dissociation of interfacial charge transfer (CT) states. Motions of electrons and holes is scrutinized separately by selectively probing the Stark shift dynamics at selected wavelengths. It is shown that only charge pairs with an effective electron-hole separation distance of less than $4 \mathrm{~nm}$ are created during the dissociation of Frenkel excitons. Dissociation of the coulombically bound charge pairs is identified as the major rate-limiting step for charge carriers' generation. Interfacial CT states split into free charges on the time-scale of tens to hundreds of picoseconds, mainly by electron escape from the Coulomb potential over a barrier that is lowered by the electric field. The motion of holes in the small molecule donor material during the charge separation time is found to be insignificant.

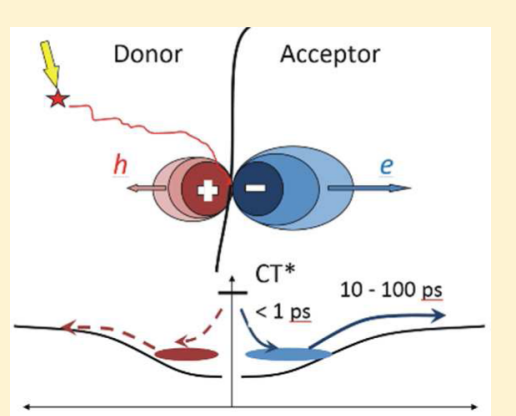

Separation distance

\section{INTRODUCTION}

Organic solar cells have recently overcome $10 \%$ efficiency ${ }^{1}$ getting this technology closer to mass production due to lower fabrication cost as compared to conventional inorganic solar cells. Successful development of the field requires a clear understanding of all optical and electronic processes that determine the operation efficiency of organic solar cells. Surprisingly, the key operation process, that is, the photogeneration of free charge carriers, is still obscured.

Generally, charge carrier generation occurs in a sequence of several distinguishable steps: (i) photon absorption leading to creation of neutral Frenkel excitons in either the donor or acceptor material, (ii) Frenkel exciton migration to the donor/ acceptor interface, (iii) charge transfer (CT) between donor and acceptor materials, (iv) formation of a coulomb bound charge pair across the heterojunction (interfacial charge transfer state), and (v) dissociation of the relaxed CT state into free charge carriers. The first three steps have been intensively investigated by means of ultrafast spectroscopy methods. It was shown that formation of the CT state from a Frenkel exciton created in the donor material is extremely fast taking place on a time scale of tens of femtoseconds. ${ }^{2-7}$ Information about the last two processes, that is, how the CT state stabilizes and splits into free charge carriers, is more controversial. A number of studies, mainly performed in bulk heterojunction solar cells, have been published during the last several years arguing for two different conceptions: According to the first one, charge carriers are generated from relaxed interfacial CT states and the separation is driven by carrier diffusion in disordered threedimensional materials. ${ }^{8-12}$ The second conception claims that charge carriers are generated on an ultrafast time scale by dissociation of hot, delocalized CT states. ${ }^{13-17}$ Investigations have indeed demonstrated that charge pairs with electron-hole separation larger than the nearest neighbor distance are created from nonrelaxed interfacial CT states. ${ }^{14,17}$ Ultrafast electric field optical probing techniques have been demonstrated as a very useful tool for the investigation of electrical charge separation and transport phenomena in organic semiconductors. ${ }^{15,18-20}$ These experimental approaches employ electric field-dependent optical response of the investigated material, be it field-induced second harmonic generation or Stark effect. Photogeneration of the charge pairs, splitting of these pairs, and subsequent carrier drift result in the modification of macroscopic and/or microscopic distributions of the electric field in the material, causing modifications in its optical properties. Tracking the temporal evolution of these properties in the optical pumpprobe scheme enables the reconstruction of the electric field and, hence, the dynamics of charge motion. Gélinas et al. ${ }^{15}$ evaluated from analysis of the Stark shift input into the

Received: April 9, 2015

Published: June 3, 2015 
transient absorption signal that charge carriers are separated by at least $4 \mathrm{~nm}$ by electron delocalization and coherent propagation inside fullerene domains. The authors considered that the remaining coulomb attraction barrier is low so that charge carriers created after this ultrafast propagation may be considered as free. However, experimentally, the subsequent charge pair evolution remained undisclosed. The $\sim 4 \mathrm{~nm}$ separation distance of the electron-hole pair is much shorter than the coulomb capture radius, estimated to be about $12-15$ $\mathrm{nm}$. We have also investigated the carrier drift dynamics in bulk heterojunction solar cells by means of the ultrafast optical probing of the electric field kinetics. ${ }^{21}$ These measurements revealed ultrafast evolution of the average carrier drift distance. The charge pair separation dynamics in these measurements, however, was convoluted with the complex three-dimensional motion of already separated charge carriers in the disordered bulk heterojunction. Formation of interfacial charge transfer states and their dissociation into free charge carriers in planar heterojunction solar cells were more rarely addressed. These processes are expected to be governed by the same physical interactions and material properties as in bulk heterojunction solar cells. Because of precisely known geometrical parameters and a well-defined geometrical plane where separation of the charges takes place, CT state dynamics and free carrier separation in bilayer solar cells are expected to be less entangled. The averaged microscopic displacement of charges at the planar heterojunction results in macroscopic perturbation of the electric field in the donor and in the acceptor layers. In addition, transport of photogenerated electrons and holes is spatially separated. Thus, planar heterojunction solar cells may serve as an ideal model system for the investigation of the charge carrier generation phenomenon.

To the best of our knowledge, the carrier escape from the Coulomb attraction and the dynamics leading to free charge carriers have not been addressed explicitly so far, probably because of the absence of appropriate experimental methods capable to disclose the carrier motion within organic photovoltaic devices with a sufficient time resolution. Here we investigate the free charge carrier formation process by use of ultrafast time-resolved Stark effect spectroscopy, which allows one to probe directly the electric field dynamics in cyanine/ fullerene bilayer solar cells. The important advantage of the Stark effect technique when applied to planar heterojunction solar cells is that it allows one to distinguish between the electron and hole motions by analyzing different spectral regions, as long as electroabsorption (EA) of the donor and acceptor materials is spectrally separated. Moreover, fast CT state formation, fast electron motion in thin fullerene layer, and close to one-directional carrier motion enabled us to distinguish between CT state formation, coulomb bound charge pair separation, and carrier drift processes. We demonstrate that charge pairs with an effective separation distance of less than 4 $\mathrm{nm}$ are created on an ultrafast time scale at the small molecule/ fullerene interface, while dissociation of coulomb bound charge pairs into free charge carriers takes place on the tens to hundreds of picoseconds time scale from the relaxed CT state mainly by the electron motion.

\section{EXPERIMENTAL SECTION}

OPV cells with planar heterojunction architecture were prepared by spin-coating a solution in 2,2,3,3-tetrafluoropropanol of the trimethine cyanine dye $\mathrm{Cy} 3$ with $\mathrm{PF}_{6}{ }^{-}$as a counterion (Cy3-P) and by vacuum thermal evaporation of $\mathrm{C}_{60}$ on top of the dye layer. The molecular structures of the investigated materials and their energy levels are shown in Figure 1. A detailed sample preparation method has been

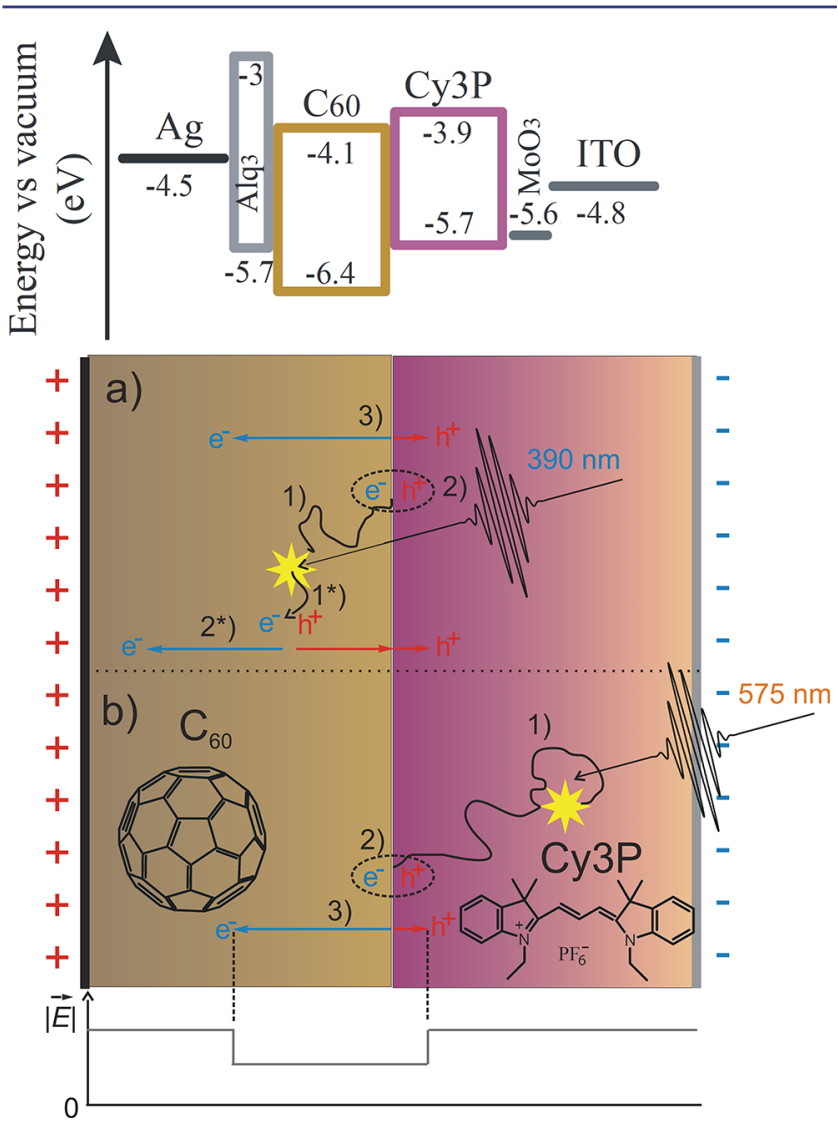

Figure 1. Energy level diagram of the solar cell (upper scheme). Schematic illustration of charge formation in a $\mathrm{C}_{60} / \mathrm{Cy} 3-\mathrm{P}$ planar heterojunction solar cell and the resulting redistribution of the electric field under pulsed excitation conditions (bottom scheme): (1) diffusion of the photogenerated exciton toward the interface; (2) formation and splitting of the interfacial CT state; (3) drift of free charge carriers. Processes marked by an asterisk refer to electric fieldassisted generation $\left(1^{*}\right)$ and drift $\left(2^{*}\right)$ of free charges in the bulk of $\mathrm{C}_{60}$. Part (a) represents the case of acceptor excitation $(390 \mathrm{~nm})$, and part (b) donor excitation $(575 \mathrm{~nm})$.

reported elsewhere. ${ }^{22}$ Such cyanine dye/fullerene bilayer organic solar cells have shown lately up to $3.2 \%$ power conversion efficiency, with a short-circuit current $J_{\mathrm{sc}}=6.5 \mathrm{~mA} \cdot \mathrm{cm}^{-2}$, an open-circuit voltage $V_{\mathrm{oc}}=$ $0.95 \mathrm{~V}$, and a fill factor $\mathrm{FF}=52.4 \% .^{23}$ The thorough analysis of the device performance can be found in ref 24 .

The experimental setup used for the electromodulated differential absorption (EDA) measurements was implemented on a conventional femtosecond absorption pump-probe spectrometer. More details can be found in ref 25 .

\section{RESULTS AND DISCUSSION}

The time-resolved Stark effect technique consists in probing the dynamics of spectral shifts in the transient absorption of a bilayer sample submitted to an external static electric field. Excitons created by a light pulse in the bulk of the cyanine Cy3$\mathrm{P}$ layer diffuse and are quenched at the donor-acceptor interface by electron transfer from the Cy3-P to the energetically lower lying LUMO of $\mathrm{C}_{60}$. Diffusion of the excitons does not affect the electric field felt by the donor material, but the electric field distribution starts being affected as electrons are injected into the acceptor layer and CT states 
are created at the interface. The effect is twofold: First, local electric fields appear with the electron injection into the acceptor layer. Second, dissociation of the CT states is followed by the drift of the formed free charge carriers toward the corresponding electrodes, and this reduces the macroscopic built-in or applied electric field. The extraction of electrons and holes reduces the field in the acceptor and donor layers, respectively (see Figure 1). The analogy may be applied for the case of acceptor excitation, except that exciton diffusion takes place in the acceptor layer and a hole is injected into the HOMO of the donor at the interface. As we show below, fieldassisted free charge generation in the bulk of the electron acceptor $\mathrm{C}_{60}$ may also significantly contribute to photocurrent if an external bias is applied to the device.

The application of this technique is based on the measurement and analysis of the time-resolved EDA spectra. The EDA is an optical pump-probe method designed to measure the absorption difference between a biased and unbiased sample as a function of probe delay. General aspects of the EDA technique were discussed in the literature. ${ }^{18,20}$

The steady-state EA spectra of pristine Cy3-P, pristine $\mathrm{C}_{60}$ and $\mathrm{C}_{60} / \mathrm{Cy} 3-\mathrm{P}(30 / 20)$ bilayer devices measured under an applied reverse bias are presented in Figure 2. Here and further

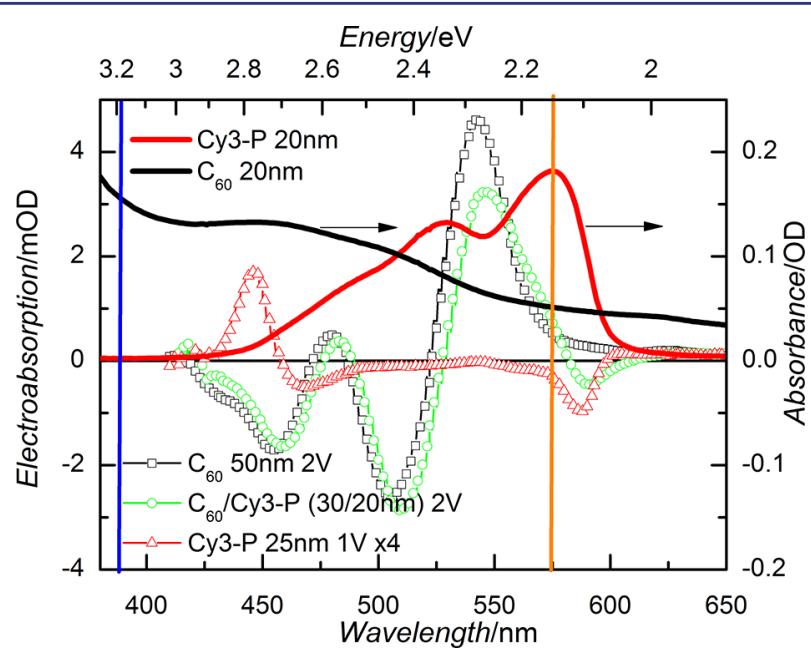

Figure 2. Electroabsorption spectra of pristine $\mathrm{C}_{60}$, pristine Cy3-P films, and $\mathrm{C}_{60} / \mathrm{Cy} 3-\mathrm{P}$ bilayer devices (data points, bias voltages, and thicknesses as indicated in the label). Absorption spectra of pristine films are also shown (right scale, full lines). Vertical lines show both 390 and $575 \mathrm{~nm}$ wavelength excitation chosen for selective excitation of $\mathrm{C}_{60}$ and Cy3-P, respectively.

in the text the label $\mathrm{C}_{60} / \mathrm{Cy} 3-\mathrm{P}(-/-)$ denotes the thickness of the corresponding layers of the device. In this case $(30 / 20)$ means that the thickness of the $\mathrm{C}_{60}$ layer is $30 \mathrm{~nm}$ and the thickness of the Cy3-P layer is $20 \mathrm{~nm}$. Dark currents under reverse bias were negligible (below $1 \mathrm{~mA} / \mathrm{cm}^{2}$ ) and, thus, charge concentration in the active layers was virtually zero. This situation results in a homogeneously distributed electric field for different layer thicknesses and in both layers of the bilayer structure. The EA spectrum of $\mathrm{C}_{60}$ displays two main features: a positive peak located at $540 \mathrm{~nm}$ and a negative one at $505 \mathrm{~nm}$. This spectrum is consistent with the one reported in literature. $^{26,27}$ Notably, the EA spectrum doesn't follow the first or the second derivative of the absorption spectrum. This is due to the forbidden optical transition from $\mathrm{A}_{\mathrm{g}}$ ground state to $\mathrm{H}_{\mathrm{g}}$ symmetry excited state that becomes allowed in the presence of the electric field. ${ }^{27,28}$ A red band at $585 \mathrm{~nm}$ and a blue band at $445 \mathrm{~nm}$ are observed in the spectra of pristine Cy3-P. Importantly, there are regions in the visible spectrum where the EA bands of $\mathrm{C}_{60}$ and Cy3-P are not overlapping. For instance, the EA amplitude of Cy3-P is zero in the range from 500 to $550 \mathrm{~nm}$, exactly where $\mathrm{EA}$ of $\mathrm{C}_{60}$ is the strongest. The red band of Cy3-P at $585 \mathrm{~nm}$ overlaps only slightly with the red tail of the $\mathrm{C}_{60}$ main peak. Spectral separation of the Cy3-P and $\mathrm{C}_{60}$ EA allows independent monitoring of changes of the electric field in each of the layers by analyzing different spectral regions in time-resolved EDA experiments. In addition, selective excitation of the donor or acceptor layer is also possible by appropriate choice of the excitation wavelength. The absorbance maximum of Cy3-P is located at $575 \mathrm{~nm}$, where the absorbance of $\mathrm{C}_{60}$ is weak. The opposite situation prevails in the near-UV where Cy3-P does not absorb, while $\mathrm{C}_{60}$ has a rather strong absorbance (Figure 2).

The steady-state amplitude of $\mathrm{C}_{60}$ EA peaks at 540 and 505 nm scale quadratically with the applied bias (inset of Figure 3a). Photoexcitation of the device, as it was discussed, causes reduction of the electric field and weakening of the EA amplitude. Electrons drifting away from the interface in the $\mathrm{C}_{60}$

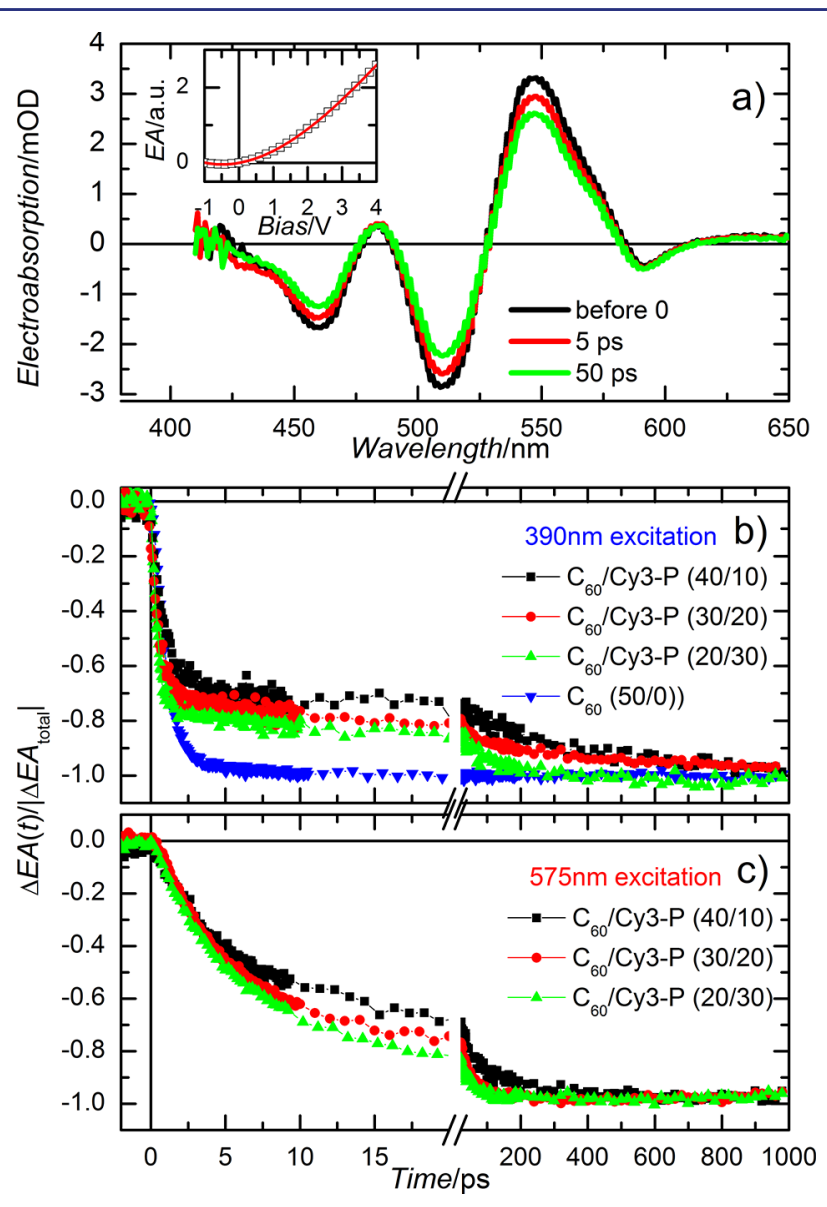

Figure 3. (a) EDA spectra of a $\mathrm{C}_{60} / \mathrm{Cy} 3-\mathrm{P}(30 / 20)$ device at different time delays after $575 \mathrm{~nm}$ excitation, $0.2 \mu \mathrm{J} / \mathrm{cm}^{2}$, for $4 \mathrm{~V}$ applied bias. The inset shows the steady-state dependence of the EA amplitude on the applied bias. Electroabsorption dynamics in the $\mathrm{C}_{60}$ layer of planar heterojunction devices of different compositions at (b) $390 \mathrm{~nm}(0.7$ $\left.\mu \mathrm{J} / \mathrm{cm}^{2}\right)$ and (c) $575 \mathrm{~nm}$ excitation wavelength $\left(0.2 \mu \mathrm{J} / \mathrm{cm}^{2}\right)$ with $2 \mathrm{~V}$ applied bias $(\sim 0.5 \mathrm{MV} / \mathrm{cm})$. Part (b) includes the electric field dynamics in a pristine $\mathrm{C}_{60}$ film. 
layer and geminate holes create electrical dipoles, which counteract the initial electric field (Figure 1). All measurements were performed at small charge drift conditions; ${ }^{29}$ that is, the excitation intensity was chosen in a way that the concentration of photogenerated carriers was too low to perturb the initial electric field significantly. Thus, the excitation-induced variation of the field was small in comparison to the applied field, and the motion of charges might be considered as taking place in a quasi-static electric field. Figure $3 \mathrm{a}$ shows the EDA spectra of the bilayer device $\mathrm{C}_{60} / \mathrm{Cy} 3-\mathrm{P}(30 / 20)$ at different delay times after excitation by a laser pulse at $575 \mathrm{~nm}$ wavelength. In general, EDA data may also include the absorption changes caused by field-affected population of the excited state(s). This contribution was negligible at the low excitation energy fluence of $0.2 \mu \mathrm{J} / \mathrm{cm}^{2}$ (Figure 3a), as no shift of the spectral bands or appearance of new band is observed. Moreover, only the $\mathrm{C}_{60}$ EA bands experience a decay in time, while the EA band at 585 $\mathrm{nm}$ attributed to Cy3-P remains constant during the optically probed time interval of $1 \mathrm{~ns}$. Thus, the major contributor to the EDA dynamics is the decaying electric field in the $\mathrm{C}_{60}$ layer related to the drift of photogenerated electrons. Apparently, the holes' drift in Cy3-P is much slower. This is in agreement with the low hole mobility of about $10^{-5}-10^{-7} \mathrm{~cm}^{2} /(\mathrm{V} \cdot \mathrm{s})$ in Cy3$\mathrm{P}^{22}$ with respect to the electron mobility of the order of $1 \mathrm{~cm}^{2} /$ $(\mathrm{V} \cdot \mathrm{s})$ in $\mathrm{C}_{60} \cdot{ }^{30-33}$ Accordingly, we expect hole motion on a microsecond time scale that is confirmed by integral mode time-of-flight data (see the Supporting Information (SI)).

Figure $3 \mathrm{~b}$ (acceptor excitation) and Figure 3c (donor excitation) show the dynamics of the EA absolute amplitude integrated in the spectral region between 500 and $560 \mathrm{~nm}$, where the main peak of the $\mathrm{C}_{60}$ electroabsorption is located. The EA changes are normalized to the total EA change $\left(\Delta \mathrm{EA}_{\text {total }}\right)$ calculated from the time-integrated photocurrent measured with the oscilloscope. The time-integrated photocurrent divided by the sample capacitance yields the total reduction of the electric field (voltage). In conjunction with the steady-state dependence of the EA versus bias, this enables one to estimate $\Delta \mathrm{EA}_{\text {total }}$, the change of $\mathrm{EA}$ when all electrons are extracted from bilayer devices or all charges from the single $\mathrm{C}_{60}$ layer device. Thus, reaching the " -1 " level indicates that all electrons (or all electrons and holes in the case of single $\mathrm{C}_{60}$ layer) were extracted from the sample-a plateau extending to 1 ns as determined from optical measurements corresponds to the same level as obtained at long times from time-integrated electrical measurements of the photocurrent which is not able to resolve the picosecond dynamics due to its limited timeresolution. The obtained electric field dynamics are qualitatively similar for different device compositions, but strongly differ depending on the selective excitation of the $\mathrm{C}_{60}(390 \mathrm{~nm}$, Figure 3b) or Cy3-P (575 nm, Figure 3c) layer.

First, we discuss results for the neat $\mathrm{C}_{60}$ device $(390 \mathrm{~nm}$ excitation, blue curve in Figure $3 \mathrm{~b}$ ). The amount of extracted charges increases almost linearly with applied bias (see the SI) for this sample. This indicates field-assisted generation of free carriers in the bulk of $\mathrm{C}_{60}$. Quite efficient carrier generation in $\mathrm{C}_{60}$ is related to the existence of CT states (within $\mathrm{C}_{60}$, not to be confused with $\mathrm{CT}$ states at the interface) located at higher energies than Frenkel exciton states. ${ }^{34}$ Thus, carrier generation in the bulk of $\mathrm{C}_{60}$ is expected to be very fast taking place before relaxation to the Frenkel exciton state. We observe a very fast decay of the EA in this sample. During the first few picoseconds, final reduction of the EA is reached. This confirms ultrafast carrier generation and indicates that carrier extraction is also very fast. Notably, we expect both, electrons and holes, to drift and be extracted on the same fast time-scale. Decay of the $\mathrm{EA}$ in pristine $\mathrm{C}_{60}$ film within a few picoseconds is consistent with mobilities of the order of $1 \mathrm{~cm}^{2} /(\mathrm{V} \cdot \mathrm{s})$ for both types of carriers. ${ }^{30,31}$ Thus, free carriers are extracted from the $\mathrm{C}_{60}$ film on the time-scale from few to several picoseconds at a field strength of about $0.5 \mathrm{MV} / \mathrm{cm}$.

Very similar EA dynamics were also observed in bilayer devices (Figure $3 \mathrm{~b}$ ) when $\mathrm{C}_{60}$ was selectively excited at $390 \mathrm{~nm}$. One can notice that the initial decay is slightly faster for the devices with thinner $\mathrm{C}_{60}$ layer. This is a consistent observation as the extraction of the charge carriers is faster from the thinner film. The main difference between the pristine $C_{60}$ and bilayer devices is the appearance of a slow component in the EA decay, which constitutes about $20-25 \%$ of the total amplitude in bilayers. We suggest that the fast component corresponds to the same field-assisted mechanism of free charge generation from CT states in the bulk of the $\mathrm{C}_{60}$ layer as in the pristine $\mathrm{C}_{60}$ device, while the slow component is attributed to the diffusion of Frenkel excitons in the $\mathrm{C}_{60}$ layer followed by charge generation at the interface between $\mathrm{C}_{60}$ and $\mathrm{Cy} 3-\mathrm{P}$. This slow decay is consistent with the relatively slow exciton diffusion in $\mathrm{C}_{60}{ }^{35}$ and the exciton lifetime of about $1.2 \mathrm{~ns}^{36-38}$ Contribution of the fast decay component, which is attributed to field-assisted charge generation, is slightly larger for the devices with thinner fullerene layer. This is explained by the redistribution of the applied field in the bilayer according to relative permittivity of each of the layers. The cyanine has by about a factor of 1.5 higher relative permittivity than the fullerene. At the same applied voltage and the same overall thickness of $50 \mathrm{~nm}$, the electric field in the fullerene layer is stronger for those devices where this layer is thinner.

The rate of the EA decay in the $\mathrm{C}_{60}$ layer is slower if Cy3-P is excited (Figure $3 \mathrm{c}$ ). In this case, free electrons in fullerene are generated only by dissociation of interfacial $\mathrm{CT}$ states created after Frenkel excitons in Cy3-P diffusionally reach the interface. The EA decay rate is determined by exciton diffusion to the interface, formation of the interfacial CT states, their splitting into pairs of free charge carriers and electron drift through the $\mathrm{C}_{60}$ layer. There are several indications that exciton diffusion in Cy3-P is not a major retarding factor. First, the decay of the electric field is virtually independent of the Cy3-P layer thickness: the diffusion time is expected to be proportional to the square of the distance, and therefore, one would expect a strong dependence. Surprisingly, the opposite was observed: the decay is the slowest in the $\mathrm{C}_{60} / \mathrm{Cy} 3-\mathrm{P}(40 / 10)$ device having the thinnest Cy3-P layer. Second, our ultrafast fluorescence measurements revealed a Cy3-P exciton lifetime in the planar heterojunction devices of the order of a few picoseconds (see the SI), indicating very fast exciton diffusion and quenching at the interface with $\mathrm{C}_{60}$, that is, much shorter than the carrier extraction. And third, the rate of the EA decay increases with applied bias (see Figure 4). Diffusion of excitons, which are neutral particles, cannot be influenced by the field. The second process, CT exciton formation, was determined to take place on a femtosecond time scale in different bulk heterojunction solar cells. Moreover the fast fluorescence decay also limits this time to several ps at most. Electron extraction rate also cannot be the main retarding factor, because this time, for the applied voltages of $1-4 \mathrm{~V}$, it remains on a few picosecond time scale, according to the result at $390 \mathrm{~nm}$ excitation (see the SI). 


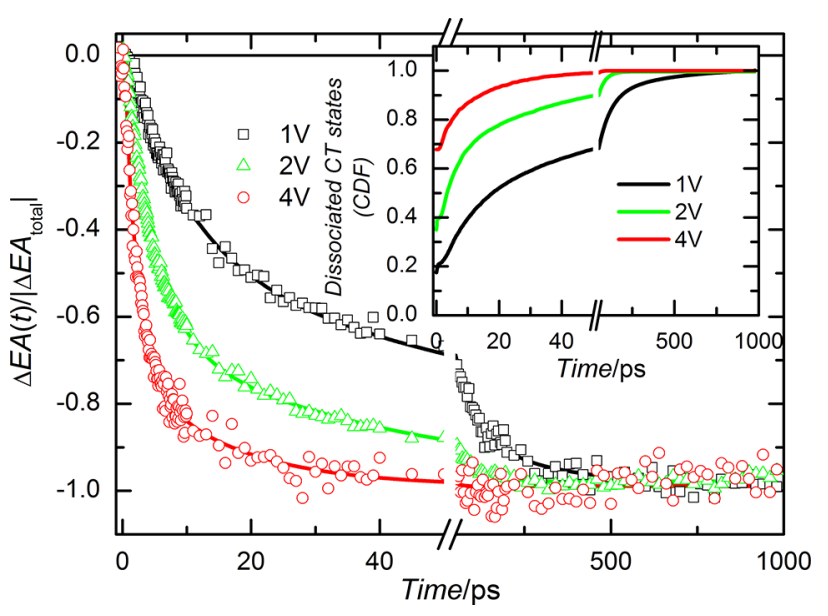

Figure 4. Electroabsorption dynamics in the $\mathrm{C}_{60}$ layer of the $\mathrm{C}_{60} / \mathrm{Cy} 3-$ $\mathrm{P}(30 / 20)$ device at $575 \mathrm{~nm}$ excitation wavelength $\left(0.2 \mu \mathrm{J} / \mathrm{cm}^{2}\right)$ and different applied biases. Data points indicate experimental data, and lines are results from modeling. The inset shows obtained cumulative distribution functions $(\mathrm{CDF})$ of the CT state lifetime $\left(t_{\mathrm{ct}}\right)$ at the corresponding biases.

These considerations lead to the conclusion that dissociation of the interfacial CT state into free charge carriers is the major rate-limiting process. Its rate depends on the applied electric field, which, apparently, lowers the energy barrier for free charge formation created by intrinsic attraction of geminate countercharges. The time scale of the decay lies in the range of tens of picoseconds, indicating that a relaxed CT state is the major precursor to free charge carriers, while contribution of vibrationally "hot" CT states to the formation of free charge carriers is minor.

The relatively slow, field-stimulated EA decay in our data implies that the electron for some time remains in the potential well once it is injected from Cy3-P into the fullerene layer. We have no information if the charge carriers during this time are localized on the nearest neighbor Cy3-P and fullerene molecules (CT state), or are separated by larger distances and delocalized to some extent in donor or acceptor domains. We refer to this state as an interfacial CT state, although, "geminate charge pair" may be a synonym.

We can set an upper limit for the effective charge separation distance in the CT state from the analysis of the EA kinetics at different applied voltages. Generally, it would be possible to reconstruct the exact motion kinetics of electrons according to the EA dynamics, but there is fundamental uncertainty at close electron-hole separation distance, were the point-charge approximation fails. Gélinas et al. considered EA as originating from the local electric fields that appear as the charges climb the potential well of Coulomb attraction, separate and gain potential energy in the form of electrostatic field. ${ }^{15}$ Notably, we did not observe any increase of EA in the bilayer and in the pristine $\mathrm{C}_{60}$ samples. Also, there was no detectable signal in the zero-field transient-absorption data at the given fluence of 0.2 $\mu \mathrm{J} / \mathrm{cm}^{2}$ (not shown), which would indicate electroabsorption originating from the local fields of CT states. Thus, we conclude that the electroabsorption caused by the CT states was negligible in comparison with the electroabsorption decay caused by drifting electrons. The monitored EA signal is proportional to the square of the electric field integrated over the probed volume $\left(\mathrm{EA} \sim \int E^{2} d \mathrm{~V}\right)$. The electric field energy is also proportional to the same integral. Therefore, the change of
EA induced by the motion of the carrier is determined by the potential in which it is moving. This potential greatly depends on the delocalization of the charges and delocalization is named among the key factors that facilitate charge separation. Figure 5

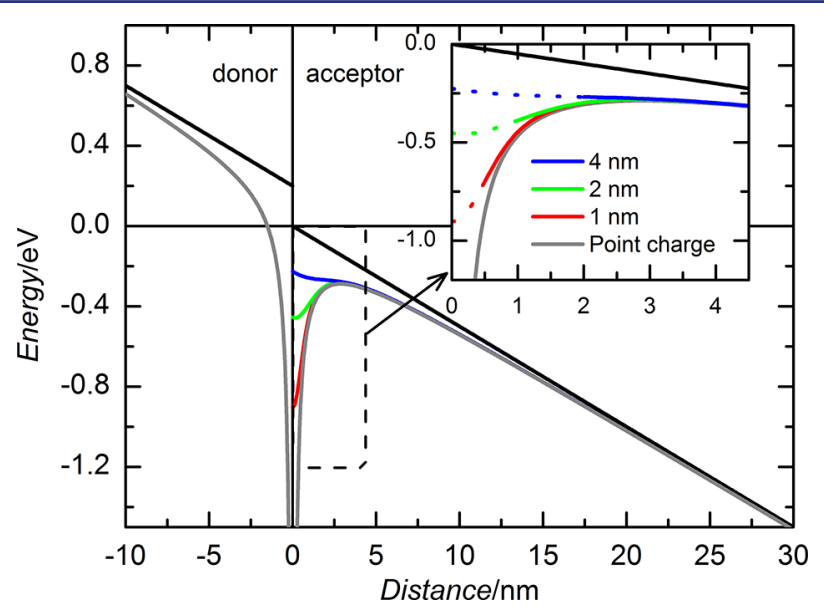

Figure 5. Hypothetic electron potential in the bilayer device at the electric field of $0.5 \mathrm{MV} / \mathrm{cm}$ (black lines). The dielectric constant of the media is $3.61^{40}$ and the zero energy is assigned to the electron at the interface without counter charge. The curves show potentials in the presence of a positive point charge at the interface and different electron delocalization, from point charge to Gaussian width of $4 \mathrm{~nm}$ (width at $1 / \mathrm{e}$ of the maximum amplitude).

shows calculated electron potentials for an electric field of 0.5 $\mathrm{MV} / \mathrm{cm}$ in the vicinity of a positive point charge for different electron delocalization degrees. The potentials of delocalized electrons were calculated assuming Gaussian distribution of the electron density. The potential profiles for three different Gaussian widths of 1,2 , and $4 \mathrm{~nm}$ are presented. It follows that the upper limit for the delocalization is of about $4 \mathrm{~nm}$, because the barrier disappears at this delocalization dimension at the applied electric field of $0.5 \mathrm{MV} / \mathrm{cm}$ (corresponds to $2 \mathrm{~V}$ of applied bias in our experiments). The fact that we do not observe any increase of the EA does not contradict the existence of the potential barrier. More likely, electrons do not stay on the potential slope for a long time. They either fall back to the close CT state (bottom of the potential well) or drift toward the electrode. As a result, the number of the electrons which may give an increase of the EA is always lower than the number of already extracted electrons, which reduce the EA. We have exploited this assumption in the modeling of CT state dissociation dynamics.

The exciton diffusion in Cy3-P and electron motion through the $\mathrm{C}_{60}$ layer are faster than the $\mathrm{CT}$ state dissociation, but nevertheless, they also contribute to the delayed EA decay. Since all three processes overlap in time, the initial decay of EA is a result of the entanglement of the exciton quenching determined by the exciton diffusion, the CT state dissociation and the electron drift. In order to disentangle these processes and to get information about the CT dissociation dynamics, we have modeled the EA kinetics relying on the available information about the exciton quenching dynamics and drift of free electrons. The model accounted for the statistically distributed CT state formation and dissociation times as well as for electron drift duration through the $\mathrm{C}_{60}$ layer. The latter was evaluated from the electroabsorption dynamics for $\mathrm{C}_{60}$ excitation as described above. The dynamics of CT state 
formation was obtained from the fluorescence decay kinetics assuming that quenching of Frenkel exciton instantaneously leads to creation of the CT state. Thus, the distribution of CT state life (dissociation) times was a single free parameter, which we obtained from the best fit of experimental and calculated electroabsorption kinetics (see the SI for more details).

The modeled electroabsorption decay is shown in Figure 4 by solid lines. The inset presents the obtained cumulative distribution function (CDF) of CT state lifetimes. If all CT states were created simultaneously, CDF could be considered as a relative number of dissociated $\mathrm{CT}$ states versus time. In reality, the concentration of CT states evolves a bit differently because the formation of the CT states is spread because of the finite duration of the initiating pump pulse and, mainly, because of the exciton diffusion. Clearly, as follows from the inset of Figure 4, the applied bias enhances the rate of CT dissociation. Only a small fraction of about $20 \%$ of CT states dissociate rapidly on the sub-picosecond time scale. The rest dissociate much slower, up to $100 \mathrm{ps}$ and later at the applied bias of $1 \mathrm{~V}$. Clearly nonexponential dissociation of the CT states reflects the intrinsic disorder of the system. The number of instantaneously dissociating CT states increases to about $40 \%$ and $70 \%$ at 2 and $4 \mathrm{~V}$ of applied bias, respectively. We relate this to the elimination of the potential barrier for electron-hole separation by the applied electric field. This experimental method cannot track the CT dissociation at low electric fields because of the limited sensitivity, but, following the observed trend, prompt dissociation is likely absent and retarded dissociation is slow at the operation conditions of the Cy3-P bilayer solar cell. The CT states existing up to hundreds of picoseconds must be quasi-equilibrated at the given temperature and electric field, thus losing the memory of initial conditions of electron injection to the acceptor layer. The conclusion is that the initial separation is not a determining factor for the splitting of the CT states. This conclusion is supported by efficient charge separation by relaxed CT states at organic interfaces demonstrated in ref 39.

\section{CONCLUSION}

In conclusion, spectral separation of electroabsorption bands of electron donating material $\mathrm{Cy} 3-\mathrm{P}$ and accepting $\mathrm{C}_{60}$ enabled independent monitoring of electric field dynamics in each material of planar heterojunction organic solar cell by means of ultrafast transient Stark shift spectroscopy. Electron and hole motion dynamics during their separation at the donor/acceptor interface and drift through the corresponding material were identified from the perturbation of the electric field by photogenerated charge carriers. Our results revealed very fast electron drift within $\mathrm{C}_{60}$ with mobility of the order of $1 \mathrm{~cm}^{2}$ / $(\mathrm{V} \cdot \mathrm{s})$ and much slower (by 5 orders of magnitude) hole drift within Cy3-P, implying that electron motion is responsible for the carrier separation. Modeling of the electroabsorption dynamics led to the conclusion that, after creation of the CT state, electrons delocalize in fullerene domains no more than 4 $\mathrm{nm}$ and their complete escape from the coulomb potential created by holes remaining at close proximity to the interface takes hundreds of picoseconds at electric fields typical for solar cell operation conditions. Thus, we show that the electron escape from the Coulomb potential is a rate-limiting process for free carrier generation and rule out "hot" CT states as a dominating pathway for charge separation. We believe that dynamics and mechanism of charge separation are qualitatively similar at donor/acceptor interfaces of bulk heterojunction small molecule/fullerene solar cells. Consequently, for efficient solar cell performance, the electron accepting material shall ensure efficient electron delocalization and its unperturbed motion at distances beyond the coulomb potential well.

\section{ASSOCIATED CONTENT}

\section{Supporting Information}

Reverse bias effect on photocurrent, fluorescence, modeling of the electric field dynamics with CT state lifetime, and integral mode time-of-flight measurement. The Supporting Information is available free of charge on the ACS Publications website at DOI: $10.1021 /$ jacs.5b03682.

\section{AUTHOR INFORMATION}

\section{Corresponding Author}

*devigy@ar.fi.lt

\section{Notes}

The authors declare no competing financial interest.

\section{ACKNOWLEDGMENTS}

This work was partly funded by the scientific exchange programme between Switzerland and the new member states of the European Union (Sciex-NMS ${ }^{\text {ch }}$ Project No.12.059). Financial support by the Swiss National Science Foundation (Grant No. 200021_144120) and the NCCR MUST is gratefully acknowledged.

\section{REFERENCES}

(1) Green, M. A.; Emery, K.; Hishikawa, Y.; Warta, W.; Dunlop, E. D. Prog. Photovoltaics: Res. Appl. 2014, 22, 701-710.

(2) Barbour, L. W.; Hegadorn, M.; Asbury, J. B. J. J. Am. Chem. Soc. 2007, 129, 15884-15894.

(3) Parkinson, P.; Lloyd-Hughes, J.; Johnston, M. B.; Herz, L. M. Phys. Rev. B 2008, 78, 115321.

(4) Hwang, I.-W.; Moses, D.; Heeger, A. J. J. Phys. Chem. C 2008, 112, 4350-4354.

(5) Piris, J.; Dykstra, T. E.; Bakulin, A. A.; van Loosdrecht, P. H. M.; Knulst, W.; Trinh, M. T.; Schins, J. M.; Siebbeles, L. D. A. J. Phys. Chem. C 2009, 113, 14500-14506.

(6) Marsh, R. A.; Hodgkiss, J. M.; Albert-Seifried, S.; Friend, R. H. Nano Lett. 2010, 10, 923-930.

(7) Cook, S.; Katoh, R.; Furube, A. J. Phys. Chem. C 2009, 113, 2547-2552.

(8) Blom, P. W. M.; Mihailetchi, V. D.; Koster, L. J. A.; Markov, D. E. Adv. Mater. 2007, 19, 1551-1566.

(9) Lee, J.; Vandewal, K.; Yost, S. R.; Bahlke, M. E.; Goris, L.; Baldo, M. A.; Manca, J. V.; Voorhis, T. V. J. Am. Chem. Soc. 2010, 132, $11878-11880$.

(10) Vithanage, D. A.; Devižis, A.; Abramavičius, V.; Infahsaeng, Y.; Abramavičius, D.; MacKenzie, R. C. I.; Keivanidis, P. E.; Yartsev, A.; Hertel, D.; Nelson, J.; Sundström, V.; Gulbinas, V. Nat. Commun. 2013, 4, 2334.

(11) Albrecht, S.; Vandewal, K.; Tumbleston, J. R.; Fischer, F. S.; Douglas, J. D.; Fréchet, J. M.; Ludwigs, S.; Ade, H.; Salleo, A.; Neher, D. Adv. Mater. 2014, 26, 2533-2539.

(12) Jailaubekov, A. E.; Willard, A. P.; Tritsch, J. R.; Chan, W. L.; Sai, N.; Gearba, R.; Kaake, L. G.; Williams, K. J.; Leung, K.; Rossky, P. J.; Zhu, X. Y. Nat. Mater. 2013, 12, 66.

(13) Gregg, B. A. J. Phys. Chem. Lett. 2011, 2, 3013-3015.

(14) Bakulin, A. A.; Rao, A.; Pavelyev, V. G.; van Loosdrecht, P. H.; Pshenichnikov, M. S.; Niedzialek, D.; Cornil, J.; Beljonne, D.; Friend, R. H. Science 2012, 335, 1340-1344.

(15) Gélinas, S.; Rao, A.; Kumar, A.; Smith, S. L.; Chin, A. W.; Clark, J.; van der Poll, T. S.; Bazan, G. C.; Friend, R. H. Science 2014, 343, 512-516. 
(16) Deibel, C.; Strobel, T.; Dyakonov, V. Phys. Rev. Lett. 2009, 103, 036402.

(17) Grancini, G.; Maiuri, M.; Fazzi, D.; Petrozza, A.; Egelhaaf, H. J.; Brida, D.; Cerullo, G.; Lanzani, G. Nat. Mater. 2013, 12, 29-33.

(18) Gulbinas, V.; Kananavičius, R.; Valkunas, L.; Bässler, H. Phys. Rev. B 2002, 66, 233203.

(19) Devižis, A.; Serbenta, A.; Meerholz, K.; Hertel, D.; Gulbinas, V. Phys. Rev. Lett. 2009, 103, 027404.

(20) Cabanillas-Gonzalez, J.; Virgili, T.; Gambetta, A.; Lanzani, G.; Anthopoulos, T. D.; De Leeuw, D. M. Phys. Rev. Lett. 2006, 96, 106601.

(21) Pranculis, V.; Infahsaeng, Y.; Tang, Z.; Devižis, A.; Vithanage, D. A.; Ponseca, C. S.; Inganäs, O., Jr.; Yartsev, A. P.; Gulbinas, V.; Sundström, V. J. Am. Chem. Soc. 2014, 136, 11331-11338.

(22) Jenatsch, S.; Hany, R.; Véron, A. C.; Neukom, M.; Züfle, S.; Borgschulte, A.; Ruhstaller, B.; Nüesch, F. J. Phys. Chem. C 2014, 118, 17036-17045.

(23) Wicht, G.; Bücheler, S.; Dietrich, M.; Jäger, T.; Nüesch, F.; Offermans, T.; Tisserant, J.-N.; Wang, L.; Zhang, H.; Hany, R. Sol. Energy Mater. Sol. Cells 2013, 117, 585-591.

(24) Jenatsch, S.; Geiger, T.; Heier, J.; Kirsch, C.; Nüesch, F.; Paracchino, A.; Rentsch, D.; Ruhstaller, B.; Véron, A. C.; Hany, R. Sci. Technol. Adv. Mater. 2015, 16, 035003.

(25) Devižis, A.; Hertel, D.; Meerholz, K.; Gulbinas, V.; Moser, J.-E. Org. Electron. 2014, 15, 3729-3734.

(26) Drori, T.; Sheng, C. X.; Ndobe, A.; Singh, S.; Holt, J.; Vardeny,

Z. V. Phys. Rev. Lett. 2008, 101, 037401.

(27) Jeglinski, S.; Vardeny, Z. V.; Moses, D.; Srdanov, V. I.; Wudl, F. Synth. Met. 1992, 50, 557-563.

(28) Kazaoui, S.; Minami, N.; Tanabe, Y.; Byrne, H. J.; Eilmes, A.; Petelenz, P. Phys. Rev. B 1998, 58, 7689.

(29) Pivrikas, A.; Sariciftci, N. S.; Juška, G.; Österbacka, R. Prog. Photovoltaics: Res. Appl. 2007, 15, 677-696.

(30) Frankevich, E.; Maruyama, Y.; Ogata, H. Chem. Phys. Lett. 1993, $214,39-44$

(31) Frankevich, E. L.; Maruyama, Y. ICSM'94, Int. Conf. Sci. Technol. Synth. Met. 1994, 425-425.

(32) Haddon, R. C.; Perel, A. S.; Morris, R. C.; Palstra, T. T. M.; Hebard, A. F.; Fleming, R. M. Appl. Phys. Lett. 1995, 67, 121-123.

(33) Kobayashi, S.; Takenobu, T.; Mori, S.; Fujiwara, A.; Iwasa, Y. Sci. Technol. Adv. Mater. 2003, 4, 371-375.

(34) Burkhard, G. F.; Hoke, E. T.; Beiley, Z. M.; McGehee, M. D. J. Phys. Chem. C 2012, 116, 26674-26678.

(35) Hedley, G. J.; Ward, A. J.; Alekseev, A.; Howells, C. T.; Martins, E. R.; Serrano, L. A.; Cooke, G.; Ruseckas, A.; Samuel, I. D. Nat. Commun. 2013, 4, 2867.

(36) Ebbesen, T. W.; Tanigaki, K.; Kuroshima, S. Chem. Phys. Lett. 1991, 181, 501-504.

(37) Gevaert, M.; Kamat, P. V. J. Phys. Chem. 1992, 96, 9883-9888.

(38) Sauve, G.; Dimitrijevic, N. M.; Kamat, P. V. J. Phys. Chem. 1995, 99, 1199-1203.

(39) Vandewal, K.; Albrecht, S.; Hoke, E. T.; Graham, K. R.; Widmer, J.; Douglas, J. D.; Schubert, M.; Mateker, W. R.; Bloking, J. T.; Burkhard, G. F.; Sellinger, A.; Fréchet, J. M. J.; Amassian, A.; Riede, M. K.; McGehee, M. D.; Neher, D.; Salleo, A. Nat. Mater. 2014, 13, 6368.

(40) Ruoff, R. S.; Tse, D. S.; Malhotra, R; Lorents, D. C. J. Phys. Chem. 1993, 97, 3379-3383. 


\title{
Supporting Information
}

\section{Dissociation of charge transfer states and carriers separation in bilayer organic solar cells - A time-resolved electroabsorp- tion spectroscopy study}

\author{
Andrius Devižis ${ }^{\mathrm{a}, \mathrm{d}}$, ${ }^{*}$ Jelissa De Jonghe-Risse ${ }^{\mathrm{a}}$, Roland Hany ${ }^{\mathrm{b}}$, Frank Nüesch ${ }^{\mathrm{b}, \mathrm{c}}$, Sandra Jenatsch ${ }^{\mathrm{b}, \mathrm{c}}$, \\ Vidmantas Gulbinas ${ }^{\mathrm{d}}$, Jacques-E. Moser ${ }^{\mathrm{a}}$. \\ ${ }^{a}$ Photochemical Dynamics Group, Institute of Chemical Sciences and Engineering, École Polytechnique Fédérale de \\ Lausanne, $\mathrm{CH}-1015$ Lausanne, Switzerland. \\ ${ }^{b}$ Laboratory for Functional Polymers, EMPA, Swiss Federal Laboratories for Materials Science and Technology, CH- \\ 86oo, Dübendorf , Switzerland \\ ' Institute of Materials, École Polytechnique Fédérale de Lausanne, CH-1015 Lausanne, Switzerland \\ ${ }^{\mathrm{d}}$ Center for Physical Sciences and Technology, Savanoriu 231, LT-0230o Vilnius, Lithuania
}

\section{A. Reverse bias effect on photocurrent and electric field dynamics at the fullerene excitation}

Figure $\mathrm{S}_{1}$ displays the bias dependence of the timeintegrated (10 $\mu$ s time span) photocurrent measured at pulsed excitation conditions. Pristine $\mathrm{C}_{60}$ and bilayer devices show the same trend if fullerene is excited (390 $\mathrm{nm}$ excitation) - the integrated photocurrent increases with bias within the investigated bias range. This implies that the charge generation mechanism is the same in both devices. The smaller absolute amplitude of photocurrent for the bilayer device in comparison to the pristine $C_{60}$ device probably is caused by the thinner $C_{60}$ layer in the planar heterojunction device, which results in weaker absorption of the excitation light. Since both devices show similar response, we conclude that the interface is not important for carrier generation at the applied external bias and that the charges must be generated in the bulk of $\mathrm{C}_{60}$. The process of charge generation changes substantially if donor $\mathrm{Cy}_{3}-\mathrm{P}$ is excited at $575 \mathrm{~nm}$. The saturation of the photocurrent is observed at about $0.5 \mathrm{~V}$ reverse bias. Evidently, this is due to the less electric field sensitive charge generation at the donor/acceptor interface than in the bulk of $\mathrm{C}_{60}$.

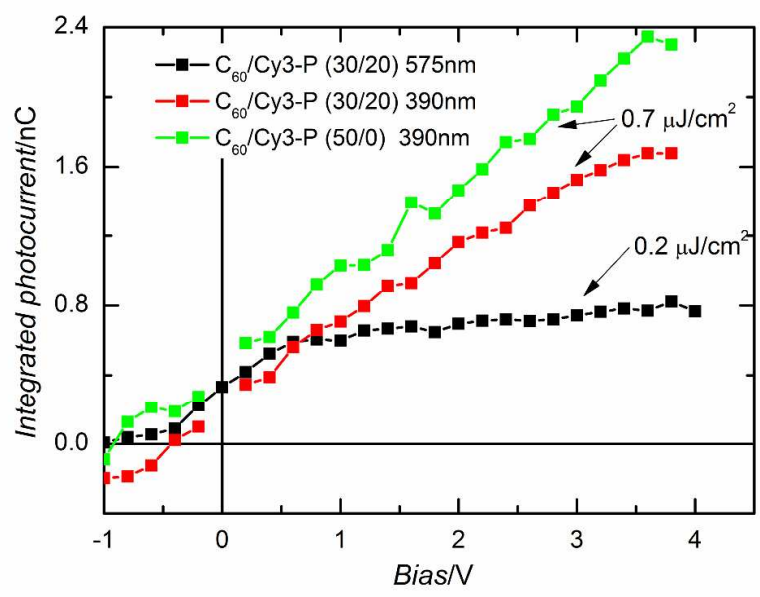

Figure S1. Integrated photocurrent as a function of applied bias for the $\mathrm{C}_{60} / \mathrm{Cy}_{3}-\mathrm{P}(30 / 20)$ bilayer and the pristine $\mathrm{C}_{60}$ film at $575 \mathrm{~nm}$ and $390 \mathrm{~nm}$ excitation.

In the main text, we have shown that the rate of the EA decay in the $\mathrm{C}_{60}$ layer slows down with decreasing bias if $\mathrm{Cy}_{3} \mathrm{P}$ is excited. We attributed this phenomenon to the increased lifetime of CT states in the weaker electric field. Obviously, the electron drift in $\mathrm{C}_{60}$ is affected by the applied bias as well. In order to support the interpretation 
it is crucial to demonstrate that bias-dependent drift of electrons is not the cause of slower EA dynamics under excitation of the donor. Direct excitation of the fullerene acceptor leads to the prompt field-assisted generation of the charge carriers in the bulk of $\mathrm{C}_{60}$. Indeed, the drift of these carriers depends on the applied bias. Figure $\mathrm{S}_{2}$ shows the decay of the electric field in $\mathrm{C}_{60}$ of the $\mathrm{C}_{60} / \mathrm{Cy3}_{3} \mathrm{P}(30 / 20)$ device at excitation wavelength of $390 \mathrm{~nm}$ and different values of applied bias. The fast decay component $(<10 \mathrm{ps})$ is attributed to the drift of promptly generated charges in the bulk of $\mathrm{C}_{60}$. As expected, it is slower at the lower applied voltage (insert of Figure S2). However, it remains much faster, few or several ps, in comparison to the EA decay under excitation of the $\mathrm{Cy}_{3}-\mathrm{P}$ (Figure 4 ). We must note that, besides the charge generation in the bulk of the $\mathrm{C}_{60}$, holes can also be injected from the electrode into $\mathrm{C}_{60}$ layer ${ }^{\left[\mathrm{S} 1, \mathrm{~S}_{2}\right]}$ Still, we consider that generation in the bulk prevails injecton from the electrode because of the following. First, it is well known that fullerene has CT states (intermolecular CT states in $\mathrm{C}_{60}$, not to be confused with interfacial CT states) which are responsible for the quite efficient charge carrier generation in pristine material (Burkhard et al. ref. 34). These CT states have higher energy than Frenkel excitons, therefore carrier generation is expected to be very fast, before relaxation to Frenkel exciton state. Second, diffusion of Frenkel excitons in fullerene is relatively slow. Hedley and co-workers (Hedley el al. ref. 35) determined that excitons reach 20$60 \mathrm{~nm}$ diameter fullerene domains during hundreds of ps. Third, fluorescence decay of single layer $C_{60}$ device is more than ten times slower (data not presented) than decay of the electric field. Therefore, carrier generation at interfaces during the exciton life-time cannot lead to the observed fast decay of the field in $\mathrm{C}_{60}$. Fourth, the long decay component in Figure $3 \mathrm{~b}$, which we attribute to the charge generation at the interface after diffusion of Frenkel excitons in $C_{60}$, becomes longer in samples with thicker $\mathrm{C}_{60}$ layer, when exciton diffusion takes longer time.

The slow decay component (Figure S2) is assigned to the charge generation at the interface, which originates from slow exciton diffusion in $\mathrm{C}_{60}$. This component, unlike the field-assisted generation in the bulk, must be less bias-sensitive. Therefore, the relative contribution of the slow component is higher at low bias. We must note that the photovoltaic performance of pristine $\mathrm{C}_{60}$ devices is poor, indicating that the electric field-assisted generation in the bulk fades to insignificant levels at the operation condition of the solar cell.

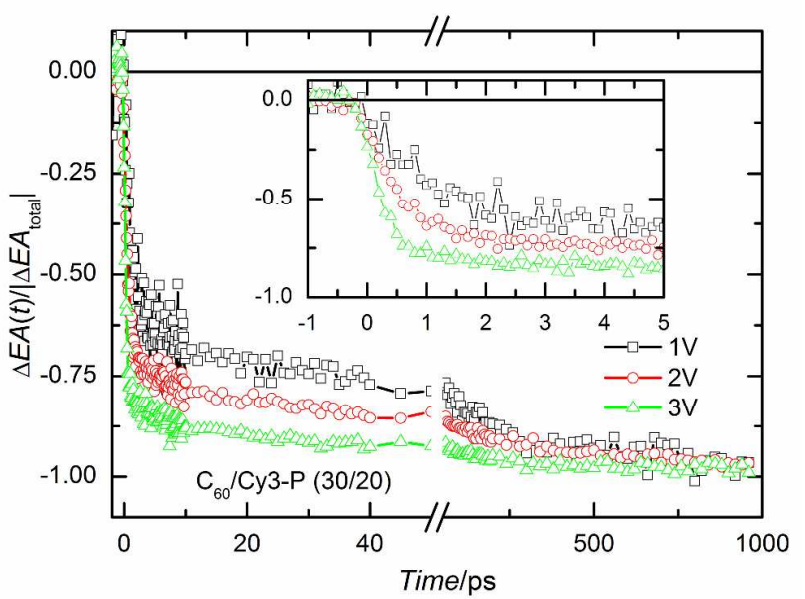

Figure $S_{2}$. Electroabsorption dynamics in the $C_{60}$ layer of $\mathrm{C}_{60} / \mathrm{Cy}_{3}-\mathrm{P}(30 / 20)$ devices at $390 \mathrm{~nm}$ excitation (0.7 $\mu \mathrm{J} / \mathrm{cm}^{2}$ ) wavelength and different applied biases. The insert shows a zoom into the fast time-scale.

\section{B. Fluorescence}

The fluorescence of a pristine Cy3-P film decays on the time-scale of tens of ps (not shown). The evolution of the exciton quenching in bilayer films was determined from the time-resolved fluorescence data measured with a streak camera. Cy3-P fluorescence in the bilayer device decays on the time-scale of few picoseconds, which is close to the time-resolution of the streak camera setup (see Figure $\mathrm{S}_{3}$ ). The actual decay of fluorescence and, thus, actual evolution of exciton concentration has to be presumed relying on the mechanism of quenching. It is unlikely that fluorescence follows an exponential decay. Excitons created in close proximity of the $\mathrm{C}_{60} / \mathrm{Cy}_{3}-\mathrm{P}$ interface are rapidly quenched, whereas more remote ones take more time to diffuse towards the interface. We assumed that the best guess for the exciton decay is the solution of the diffusion equation $\left(\frac{\partial c}{\partial t}=-D \frac{\partial^{2} c}{\partial x^{2}}\right.$, here $c$ and $D$ denote exciton concentration and diffusion coefficient) with constant initial concentration and zero concentration at the interface as the boundary condition. The numerical solution of this equation is shown in the insert of Figure $S_{3}$. The time scale is chosen in a way to give the best correspondence between the convolution of the Gaussian apparatus function with suggested exciton decay and raw experimental data. 


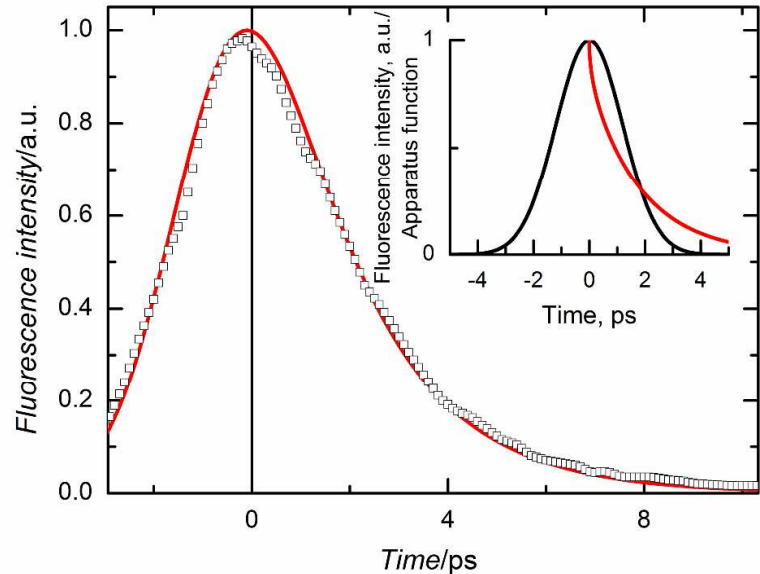

Figure S3. The fluorescence decay of a $\mathrm{C}_{60} / \mathrm{Cy}_{3}-\mathrm{P}$ (30/20) device at $600 \mathrm{~nm}$ emission wavelength (excitation wavelength $515 \mathrm{~nm}$ ). Data points represent streak camera data. Solid line show convolution of the Gaussian apparatus function with the diffusion-limited decay, which are presented in the insert (black and red, respectively).

\section{Modeling of the electric field dynamics with CT state} lifetime

The splitting of the exciton at the donor/acceptor interface determines the beginning of the electron existence in the $\mathrm{C}_{60}$ layer. The hole in $\mathrm{Cy}_{3}-\mathrm{P}$ and the electron in $\mathrm{C}_{60}$ are formed at this time $t_{e x}$. This CT state exists for the duration $t_{c t}$. Eventually, the CT state splits, the free electron drifts towards the electrode and is extracted from the $\mathrm{C}_{60}$ layer within a duration $t_{\text {drift }}$. We consider that $t_{\text {drift }}$ and the distribution of $t_{e x}$ are determined experimentally by independent measurements. Statistical distribution of $t_{e x}$ is obtained from the time-resolved fluorescence data, as described above. The drift is treated to be dispersionless, i.e. all electrons are extracted within the same duration $t_{d r i f t}$. This duration is estimated according to the field dynamics in $\mathrm{C}_{60}$ layer under direct excitation of $\mathrm{C}_{60}$ (Figure $\mathrm{S}_{2}$ ), when prompt field-assisted generation creates free carriers in the bulk of the layer. If a long-lived CT state is formed, its dissociation event should not depend on the lifetime of the initiating exciton, i.e. $t_{e x}$ and $t_{c t}$ must be independent. However, one may expect correlated $t_{e x}$ and $t_{c t}$ if fast dissociation from the "hot" states occurs. A specific computer code with a feedback loop was designed to generate optimal distribution of $t_{c t}$. The computer algorithm samples $t_{e x}$ and $t_{c t}$. The $t_{e x}$ values are generated according to the fluorescence decay (red curve, insert of Figure $\mathrm{S}_{3}$ ). Inverse transform sampling method is used. $^{\left[\mathrm{S}_{3}\right]}$ The generation of $t_{c t}$ values is not straightforward. Empirical feedback is applied in the loop procedure. The loop may be described as follows:
1) Generation of the $t_{e x}^{i}$ using inverse transform sampling (index $i$ represents iteration number).

2) Generation of $t_{c t}^{i}$ (includes comparison between the electroabsorption dynamics and the current result of the modeling at iteration $i$ )

3) Calculation of the resulting electroabsorption decay on the basis of accomplished loop iterations (all $t_{e x}^{i}, t_{c t}^{i}$ and constant $t_{\text {drift }}$ ).

4) The loop ends when $i$ reaches the predetermined number (we used 10'ooo iterations in order to get reliable statistics).

The calculation of the electroabsorption, on the basis of the obtained time values $t_{e x}, t_{c t}$ and $t_{d r i f t}$, was done assuming that the free electron drifts at the constant speed determined by the applied bias. The electron motion at close electron-hole separation, where the potential is unknown, was neglected in the evaluation of EA change. In other words, we do not account for EA originating from the $\mathrm{CT}$ states, only the drifting free electrons are considered as important for the EA decay. This simplification is supported by the fact that we do not observe increase of the EA.

We have used a discrete time-scale of 100 fs in these calculations. We must note that this procedure doesn't give an exact solution of the unknown distribution of $t_{c t}$. However, the reliability of the result may be qualitatively evaluated.

\section{Integral mode time-of-flight measurements}

The integral mode time-of-flight measurements of different devices were performed with an oscilloscope on a $10 \mathrm{k} \Omega$ load resistance. Figure $\mathrm{S}_{4}$ shows the obtained results for a $\mathrm{C}_{60} / \mathrm{Cy}_{3}-\mathrm{P}(10 / 40)$ device and a pristine $\mathrm{C}_{60}$ film. The rise-time of integrated photocurrent in the pristine $\mathrm{C}_{60}$ device (Figure $\mathrm{S}_{4} \mathrm{~b}$ ) is limited by the time resolution of the setup, which is of about $10 \mathrm{~ns}$. Consequently, the dynamics of charge extraction and bias dependences of these dynamics are not resolved by electrical measurements. The plateau extending from the $10 \mathrm{~ns}$ to microsecond time-scale is a continuation of the plateau revealed by optical probing from several ps up to 1 ns (see main text Figure $3 \mathrm{~b}$ ). This result confirms that charge carriers are extracted from $C_{60}$ film very rapidly. The situation is completely different in the bilayer device. The photocurrent evolves from early time to tens of microseconds and its dynamics is bias dependent (Figure $\mathrm{S}_{4} \mathrm{a}$ ). In the bilayer, a fast time-resolution limited rise is also observed. We attribute it to the extraction of the electrons from $C_{60}$ layer, whereas the slower dynamics should correspond to the extraction of the holes from the Cy3-P layer. The integrated photocurrent saturates after tens of microseconds indicating complete extraction of the holes at the bias of $1.2 \mathrm{~V}$. At lower biases, extraction of the holes takes more time than the investigated timeframe. 


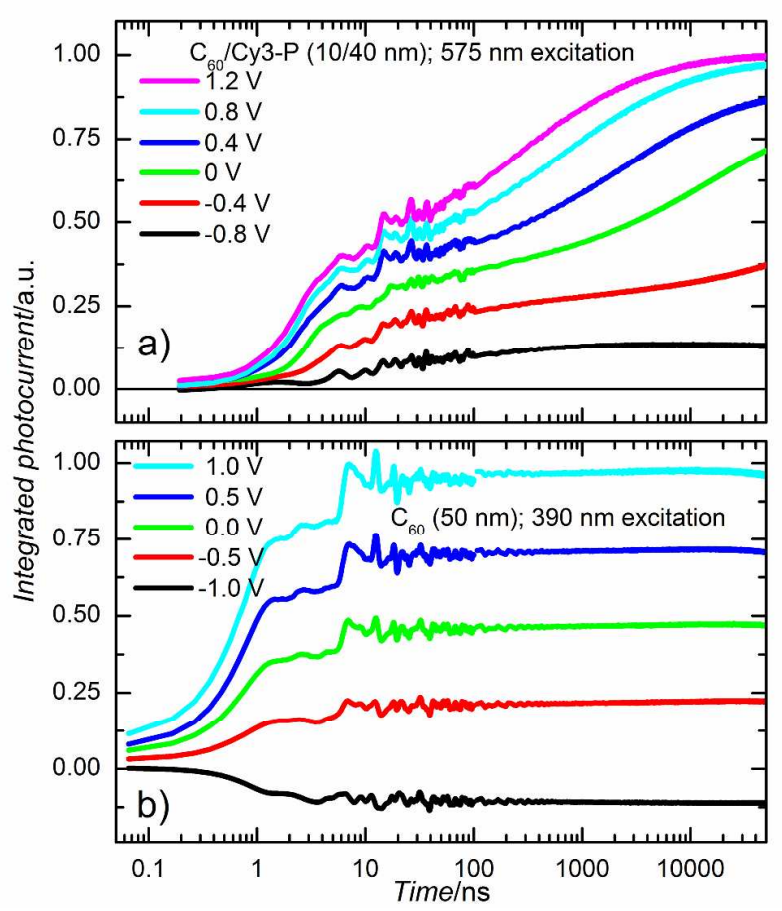

Figure S4. Integrated photocurrent versus time obtained by integral mode time of flight measurement; a) $\mathrm{C}_{60} / \mathrm{Cy3}_{-\mathrm{P}}(1 \mathrm{0} / 40), 575 \mathrm{~nm}$ excitation $\left.\left(0.2 \mu \mathrm{J} / \mathrm{cm}^{2}\right) ; \mathrm{b}\right)$ pristine $C_{60}$ film, $390 \mathrm{~nm}$ excitation $\left(0.7 \mu \mathrm{J} / \mathrm{cm}^{2}\right)$. Labels indicate different bias.

\section{References}

(S1) Mort, J.; Machonkin, M.; Ziolo, R.; Chen, I. Appl. Phys. Lett, 1992, 61, 1829-1831.

(S2) Tress, W.; Leo, K.; Riede, M. Phys. status solidi (RRL), 2013, 7, 401-405.

(S3) Devroye. L. Non-Uniform Random Variate Generation; Springer-Verlag: New York, 1998.

\section{AUTHOR INFORMATION}

\section{Corresponding Author}

*E-mail: devigy@ar.fi.lt. 